\title{
Diffuse melanosis cutis secondary to metastatic malignant melanoma: Case report
}

\section{Cheymae Saadani Hassani', Salim Gallouj', Safae Zinoune', Ghita Senhaji', Hanane Baybay', Fatima Zahra Mernissi ${ }^{1}$, Samia Arifi' ${ }^{2}$, Naoufal Mellas ${ }^{2}$}

\author{
${ }^{1}$ Departement of Dermatology, CHU Hassan II, Fès, Morroco, ${ }^{2}$ Departement of Oncology, CHU Hassan II, Fès, Morroco
}

Corresponding author: Dr. Cheymae Saadani Hassani, e-mail: ch.saadani@gmail.com

\begin{abstract}
Diffuse melanosis cutis (DMC) is an exceptional presentation of metastatic melanoma characterized by progressive bluegray discoloration of the skin and mucous membranes. It attests a generally advanced metastatic tumor extension. We report an additional case of diffuse melanosis associated with widespread cutaneous and visceral metastases from malignant melanoma. The prognosis are poor in patients with diffuse cutaneous melanosis associated with malign melanoma.
\end{abstract}

Key words: Diffuse melanosis cutis - melanoma - metastatic

\section{INTRODUCTION}

Diffuse melanosis cutis (DMC) is an exceptional presentation of metastatic melanoma characterized by progressive blue-gray discoloration of the skin and mucous membranes [1]. It attests a generally advanced metastatic tumor extension. The discoloration represents the abnormal presence of pigment within the dermis causing scattering of light [1], and is usually more intensive on sun-exposed areas, including head and upper torso [2]. Usually, dark urine associated with melanüri, darkening of the serum and peritoneal fluid can be also seen [3].

Histological findings are relatively uniform in DMC with the most consistent feature reported being the presence of dermal pigment [1].

Due to the rarity of the observed phenomenon the pathogenesis of DMC is yet to be fully understood.

In the literature, approximately 72 cases of DMC have been described until now, most of them being associated with multiple organ metastases [2].

Patients with melanosis cutis have a bleak prognosis with survival times of only weeks or months after the onset of melanosis [4].
We report an additional case of diffuse melanosis associated with widespread cutaneous and visceral metastases from malignant melanoma

\section{CASE REPORT}

A 32-year-old woman, phototype IV (Fig. 1), with history of familial melanomas, had been operated for non-metastatic melanoma of the left thigh. Five years later, the patient presented a local recurrence of her melanoma with homolateral ganglionic involvement. The results of the extension showed cutaneous, subcutaneous, ganglionic and pulmonary metastases. The patient had palliative surgery associated with chemotherapy with dacarbazine followed by paclitaxel. The evolution was marked by a change of phototype with diffuse pigmentation. The clinical examination found a patient with phototype $\mathrm{V}$ (Figs. la and lb), a diffuse and homogeneous melanoderma which interest the skin, the nails and the mucous membranes, more pronounced in photo exposed zones (Figs. 2a, 2b and 2d). Dermoscopy showed an homogeneous pigmented pattern (Fig. 2c), with diffuse regular melanonychic bands on all the nails (Fig. 2e). A skin biopsy, taken from his face area revealed melanin deposition in the dermis. Urine analysis showed a brown-amber color

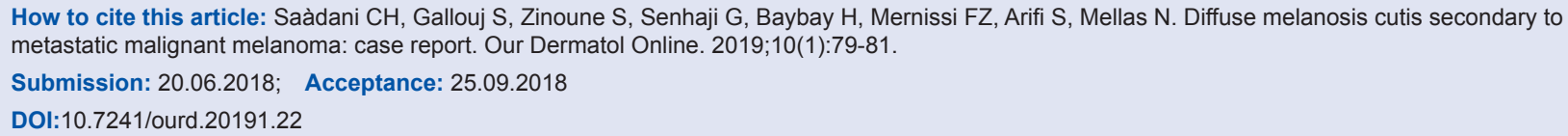


(Fig. 3). The metabolic and endocrine blood tests were negative. The patient was dead 15 months later.

\section{DISCUSSION}

Diffuse cutaneous melanosis (DCM) is rarely encountered condition in malign melanoma. It is often associated with liver, lung and lymph nodes metastases. DCM also can be seen in Addison disease, porphyria cutanea tarda and hemochromatosis [3].

Until 2017, more than 70 cases of DMC were reported, however, mechanisms of the condition have not been elucidated [2]. So far, Different pathogenic mechanisms leading to diffuse melanosis have been discussed.

Bohm et al suggested that cutaneous diffuse melanosis is linked to an excessive production of melanocytic growth factors (MSH (melanocyte stimulating hormone), Hepatocyte growth factor (HGF), Endothelin-l (ET-1)). Excessive production of MSH (from tumor), HGF and ET-1 (from distinct site of metastasis) induce normal and malignant melanocytes resulting in enhanced proliferation, melanogenesis and melanin releasing [3] .

Other several lines of evidence suggest that the direct cause of the skin darkening is melanin derived from the melanoma micrometastases in the dermis [2].

On the other hand, other reports indicate that it is plausible that cytolytic melanoma deposits liberate vast quantities of melanin precursors, as well as free melanin and melanosomes into the circulation. This may occur as a result of rapid turnover of neoplastic deposits, ischemia, immunological responses, or oncologic therapies [1]. Circulating precursors would have the capacity to traverse the glomerulus and be excreted in the urine, causing melanuria [1-6].

Subsequently, an other hypothesis is the peripheral oxidation of melanin precursors. These precursors generated in the tumor pass through the capillary membrane into the dermis and are converted into melanin by oxidizing systems 4]. This theory has been supported by the documented observations of melanin precursors, melanin, melanosomes, and melanophages in patients' serum [2].

In our patient as well, diffuse melanosis appeared after beginning chemotherapy.

The findings of histomorphological examinations are described differently [4]. The most often described

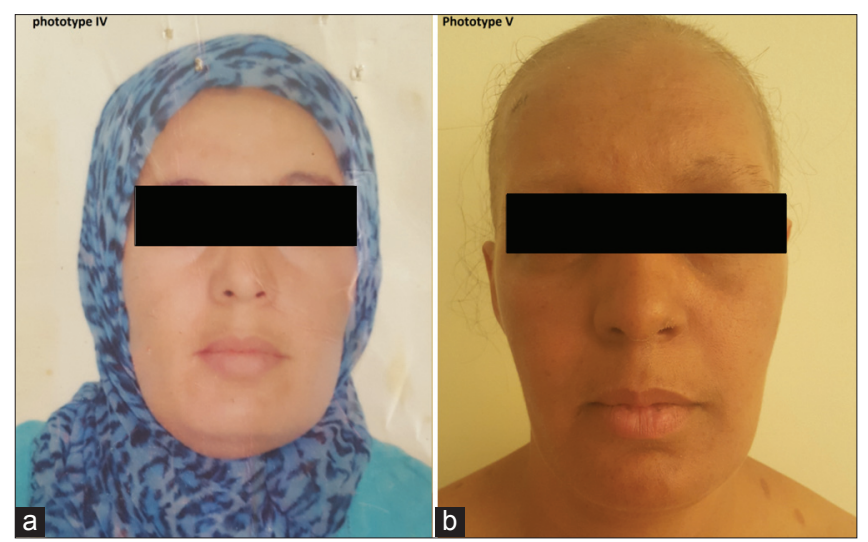

Fig. 1: Phototype of the patient before and after disease.

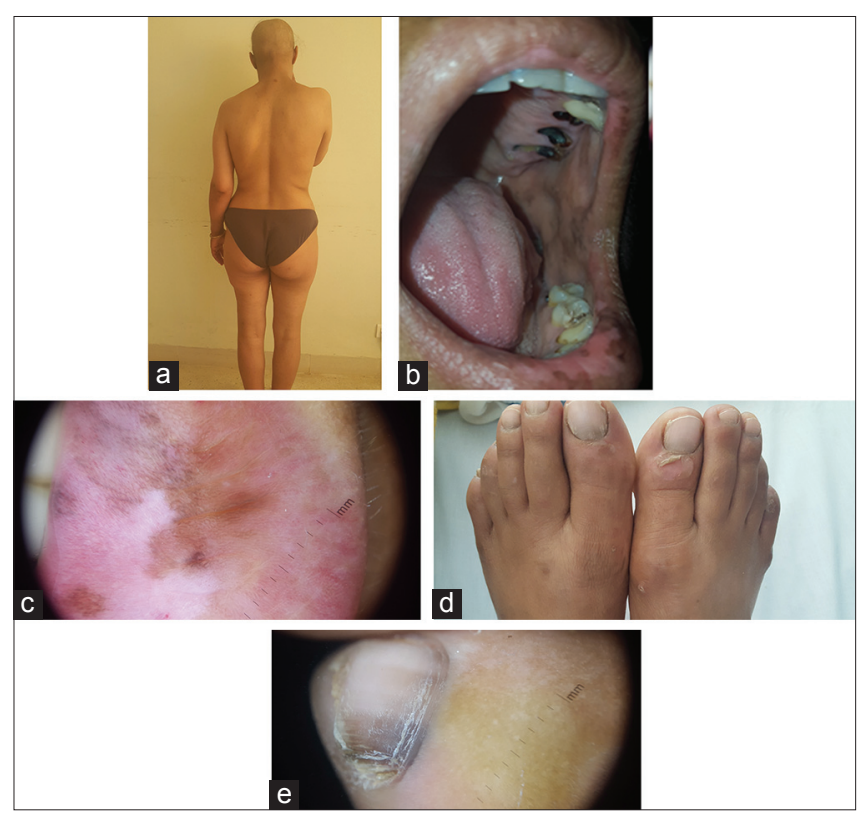

Fig 2 : a: Diffuse and homogeneous melanoderma which interest the skin, more pronounced in photoexposed zones b: Diffuse and homogeneous melanoderma which interest the mucous membranes c: Dermoscopy showed an homogeneous pigmented pattern d: Diffuse and homogeneous melanoderma which interest the nails e: Dermoscopy showed an homogeneous pigmented pattern, with diffuse regular melanonychic bands on all the nails.

histological finding, was the presence of pigmented granules but not of malignant cells [5]. In most of the cases, biopsy specimens revealed that the hyperpigmentation resulted from a marked deposition of pigment in the dermis, Another finding was giant melanosomes in melanocytes, keratinocytes and melanophages $[3,4]$. Micrometastases or single metastatic melanoma cells, as rarely have been described.

Treatment of diffuse melanosis cutis involves treating the underlying melanoma. There have been no reports of diffuse melanosis cutis reversing [7]. 


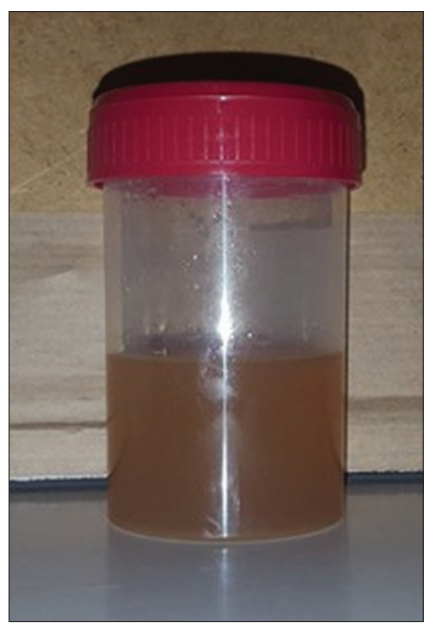

Fig 3: Urine analysis showed a brown-amber color

The prognosis are poor in patients with diffuse cutaneous melanosis $[3,4]$. The mean duration between diagnosis of melanoma and the onset of melanosis is less than a year. And the survival time from the onset of DMC is approximately 4 months [2].

DCM should be considered in the differential diagnosis list of skin discoloration after excluding other common differential diagnoses.

\section{CONCLUSION}

Melanosis cutis is a rare event in advanced metastatic melanoma whose exact mechanisms of development are still unclear. However, the role of micrometastases, as well as melanin precursors, released during lysis of MM metastases, and growth factors play a role. It is associated with a grim and poor prognosis of preterminally affected patients with a mean survival of approximately 4 months

\section{CONSENT}

The examination of the patient was conducted according to the Declaration of Helsinki principles.

\section{REFERENCES:}

1. Sebaratnam DF, Venugopal SS, Frew JW, McMillan JR, Finkelstein ER, Martin LK, et al. Diffuse melanosis cutis: A systematic review of the literature. J Am Acad Dermatol. 2013;68:482-8.

2. Maj J, Jankowska-Konsur A, Gruber J, Woźniak Z, Nockowski P, Hryncewicz-Gwóźdź A. Diffuse melanosis cutis related to dermal micrometastases as the first clinical symptom of distant metastatic malignant melanoma. Medicine (Baltimore). 2017;96:e6470.

3. Oruç, Kaplan MA, Yerlikaya H, Urakçı Z, Küçüköner M. A metastatic malign melanoma case with diffuse cutaneous melanosis. J Oncol Scien. 2016;2:71-2.

4. Hofmann M, Kiecker F, Audring H, Grefer K, Sterry W, Trefzer U. Diffuse Melanosis cutis in Disseminated Malignant Melanoma. Dermatology. 2004;209:350-2.

5. El Hadj OE, Bouhajja L, Goucha A, Rekik W, El May A, Gamoudi A. Dubreuilh's melanosis or malignant lentigo. Our Dermatol Online. 2017;8:231-2.

6. Guérin M, Chappard D, Giard C, Dompmartin-Blanchière A, Martin L. Mélanose et mélanurie au cours d'un mélanome multimétastatique. Imag Dermatol. 2012;5:1.

7. Minocha R, Kefford R, Uribe P, Sebaratnam DF, Fernandez-Penas P. Diffuse melanosis cutis in the setting of BRAFV600E mutant melanoma and treatment with targeted therapies. Australas J Dermatol. 2015;56:128-30.

Cheymae Saadani Hassani, et al. This is an open-access article distributed under the terms of the Creative Commons Attribution License, which permits unrestricted use, distribution, and reproduction in any medium, provided the original author and source are credited. Source of Support: Nil, Conflict of Interest: None declared. 